\title{
Unity through diversity in neuroscience: A journey into Scopus indexation
}

\author{
King-Hwa Ling ${ }^{1, *}$, Noraishah Mydin Abdul-Aziz ${ }^{2}$ and Norshariza Nordin ${ }^{1}$ \\ ${ }^{1}$ Department of Biomedical Sciences, Faculty of Medicine and Health Sciences, Universiti Putra Malaysia, 43400 \\ UPM Serdang, Selangor, Malaysia. \\ ${ }^{2}$ Department of Parasitology, Faculty of Medicine, Universiti Malaya, 50603 Kuala Lumpur, Malaysia. \\ * Correspondence: Ikh@upm.edu.my; Tel.: +603-9769 2564
}

Published: 30 June 2021

https://doi.org/10.31117/neuroscirn.v4i2.89

Keywords: neuroscience; neuromarketing; neuroeducation; neuroeconomic; neurolinguistics; neuropsychology; clinical neurosciences; neuropsychiatry

(C2021 by Ling et al. for use and distribution in accord with the Creative Commons Attribution (CC BY-NC 4.0) license (https://creativecommons.org/licenses/by-nc/4.0/), which permits unrestricted non-commercial use, distribution, and reproduction in any medium, provided the original author and source are credited.

Neuroscience Research Notes (ISSN: 2576-828X) was established in 2018 by a group of neuroscientists out of frustration and struggle to pay off any article processing charges for open access publication. Ever since its establishment, the journal has been steered to cater to high quality, short research and technical reports in all aspects of the nervous system (Ling et al., 2018). The journal emphasises hypothesis formation, research methodology, data interpretation and conclusion derived from both positive and negative findings, orphaned studies or neglected observations of related research fields. To date, the journal has received 66 submissions, with a $27 \%$ rejection rate. The average number of days for an editor to reach the first decision to accept any manuscript for further peer-reviewing is 5-day. However, it takes about 80-98 days (3 months) from submitting an article to final publication or rejection. The timeline of publishing with Neuroscience Research Notes is considered competitive and reasonable in fulfilling authors' interest in having their research published as soon as possible while safeguarding the sanctity of the scientific peerreviewing process.

In May 2020, the editorial board decided to submit the journal to Scopus Content Selection and Advisory Board (CSAB) for Scopus indexation. On 30 October 2020, CSAB recommended that Scopus indexes all articles published within the journal starting from 2018. The journal is indexed broadly under Neuroscience subject areas such as neurology, cognitive neuroscience, neuroscience (miscellaneous) and cellular and molecular neuroscience. The journal CiteScore for 2020 was 0.8 ( 13 citations out of 17 indexed articles) and was ranked the best under Neuroscience: Neurology subject area at position $129^{\text {th }}$ among 156 titles ( $17^{\text {th }}$ percentile) (Figure 1) (Scopus preview - Scopus - Neuroscience Research Notes, n.d.). Neuroscience Research Notes outranks several established journals in the field. It is deemed well-performed for a young journal trying to establish itself as a non-profit platinum open-access journal in a broad area of neuroscience. We expect the citations of its published articles to continue to soar and improve the CiteScore in the coming years, hence rising as a reputable neuroscience journal from scientists to scientists.

In 2021, the journal diversifies into a broader scope of neuroscience. Articles published by the journal were subcategorised into eight different subspecialties, such as:

- Neurodegeneration, Neuroinflammation and Neurotherapy,

- Neuropharmacology, Toxicology and Natural or Synthetic Products Research, 
Neuroscience Research Notes

Scopus coverage years: from 2018 to Present

Publisher: Neurotak Publishing

E-ISSN: 2576-828X

Subject area: Neuroscience: Neurology (Neuroscience: Cognitive Neuroscience

Neuroscience: Cellular and Molecular Neuroscience

Source type: Journal

Improved CiteScore methodology

Cltescore 2020 counts the citations recelved In 2017-2020 to articles, revlews, conference papers, book chapters and data

papers publlshed In 2017-2020, and divides this by the number of publications publlshed In 2017-2020. Learn more >

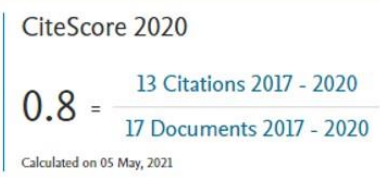

CiteScoreTracker 2021

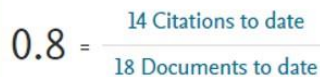

CiteScore rank 2020 ()

\begin{tabular}{|c|c|c|c|}
\hline Category & Rank & k Percentlle & \\
\hline Neurosclence & & & s \\
\hline$\llcorner$ Neurology & $\# 129 / 156$ & $=17$ th & \\
\hline Neurosclence & & & \\
\hline L Cognitive Neurosclence & \#84/96 & - 13th & \\
\hline Neurosclence & & & \\
\hline L Neurosclence (miscellaneous) & $\# 23 / 24$ & 6th & $\nabla$ \\
\hline
\end{tabular}

Figure 1: Scopus preview of Neuroscience Research Notes and its related CiteScore and rankings.

- Fundamental, Developmental and Behavioural Neurosciences,

- Neuroinformatic and Computational Neurosciences,

- Neurology, Neurosurgery and Clinical Neurosciences,

- Neuropsychiatry, Neuropsychology and Cognitive Neurosciences

- Rehabilitation \& Sport Neurosciences, and

- Neuromarketing, Neuroeducation, Neuroeconomics and Neurolinguistics.

Each section is led by 1-2 section chief editors (https://neuroscirn.org/category/), and they are empowered with more editorial responsibilities to steer their respective subspecialties forward under the auspicious Neuroscience Research Notes journal. Of all the subspecialties, the social neurosciences subspecialty is the latest addition to the journal. Social neurosciences encompassing four significant fields including neuromarketing, neuroeducation, neuroeconomics and neurolinguistics. The new wing of the journal opens up a diverse opportunity for social scientists who work with tools and datasets related to human brain activity, neurochemistry and behaviour to understand learning, language processing and interpretation, decision making, marketing trends, economic perceptions, product choices and pattern of preferences. The list is non-exhaustive and social neurosciences will bridge various fundamental and applied neurosciences to innovate applications and solve real-world problems. New fields of neuroscience 
are being explored, such as neural technology, nutritional neuroscience and space neuroscience. The inclusion of new subspecialties into the journal will make the journal unique at the forefront of unifying the multifaceted and diversified fields of neuroscience.
Acknowledgements: K.H.L is a recipient of 2021 IBRO Return Home Fellowship and ISN-CAEN Return Home Grant.

Conflicts of Interest: The authors declare no conflict of interest.

\section{References}

Ling, K. H., Abdul-Aziz, N. M., \& Nordin, N. (2018). Free open access to liberate and unleash neglected science. Neuroscience Research Notes, 1(1), 1-2. https://doi.org/10.31117/neuroscirn.v1i1.4

Scopus preview - Scopus - Neuroscience Research Notes. (n.d.). Retrieved June 30, 2021, from https://www.scopus.com/sourceid/21101038738 